05

\title{
Определение модулей упругости третьего порядка в образцах полистирола, изготовленных по разным технологиям
}

\author{
(C) А.В. Белашов, ${ }^{1}$ А.А. Жихорева, ${ }^{1}$ Я.М. Бельтюков, ${ }^{1}$ О.А. Москалюк, ${ }^{1,2}$ И.В. Семенова ${ }^{1}$ \\ ${ }^{1}$ Физико-технический институт имени А.Ф. Иоффре РАН, \\ 194021 Санкт-Петербург, Россия \\ ${ }^{2}$ Санкт-Петербургский государственный университет промышленных технологий и дизайна, \\ 191186 Санкт-Петербург, Россия \\ e-mail: irina.semenova@mail.ioffe.ru
}

Поступило в Редакцию 2 февраля 2021 г.

В окончательной редакции 16 марта 2021 г.

Принято к публикации 17 марта 2021 г.

\begin{abstract}
Ультразвуковым методом проведены измерения модулей упругости третьего порядка образцов из полистирола, изготовленных по разной технологии: двух образцов сополимера стирола с диметакрилатом этиленгликоля в разной концентрации и двух лабораторных образцов, полученных по расплавной технологии в разных температурных режимах. Показано, что зависимости нелинейных модулей упругости от частоты ультразвуковых волн в диапазоне $1-3 \mathrm{MHz}$ существенно различаются у образцов сополимера стирола и лабораторных образцов полистирола. Значения модулей лабораторных образцов, полученных по одной и той же технологии, но в разных температурных режимах, также заметно отличаются. Таким образом, технология изготовления может оказывать существенное влияние на нелинейно-упругие свойства полимерного материала.
\end{abstract}

Ключевые слова: полимеры, полистирол, нелинейные модули упругости.

DOI: 10.21883/JTF.2021.08.51094.22-21

\section{Введение}

Полимерные материалы находят все более широкое применение во многих областях промышленности, что обусловлено их высокой эластичностью, небольшой плотностью, возможностью переработки и простотой производства деталей разной формы. В то время как основные механические характеристики большинства полимерных материалов на сегодняшний день достаточно хорошо изучены, мало исследованными остаются их нелинейные упругие свойства [1]. В то же время параметры, характеризующие эти свойства, связаны с рядом важных конструктивных характеристик материалов и могут быть использованы для описания термоупругих свойств, термического старения и прогнозирования усталостных повреждений [2,3].

Для описания упругих свойств материалов используется набор параметров - модулей упругости, являющихся коэффициентами в степенном разложении потенциальной энергии по инвариантам тензора деформации. При этом в линейном приближении зависимость напряжений от деформаций описывается набором из двух модулей упругости второго порядка (например, модулем Юнга $E$ и коэффициентом Пуассона $v$, или модулями Ламе $\lambda$ и $\mu$ ), а для учета нелинейности вводится набор модулей упругости третьего порядка. Наиболее часто используются наборы модулей третьего порядка, введенные в теоретических моделях Ландау и Лифшица [4], Терстона и Браггера [5] или Мурнагана [6]. При этом наборы модулей, определенные в этих моде- лях, взаимосвязаны и могут быть пересчитаны из одного набора в другой.

На сегодняшний день разработано несколько подходов для экспериментального исследования нелинейных упругих свойств материалов и определения их нелинейно-упругих модулей, которые основаны на анализе рассеяния Мандельштама-Бриллюэна [7], параметров солитонов продольной деформации [8], волн Лэмба [9] или Релея [10], а также зависимости скоростей продольных и поперечных ультразвуковых волн в образце от приложенного давления. Последний подход был впервые предложен Хьюзом и Келли [11], и впоследствии применялся для определения нелинейных модулей упругости разных материалов: металлов и сплавов [12-14], полупроводников [15,16], горных пород [17], композитных материалов $[14,18]$ и пр. В настоящей работе этот подход использовался для определения нелинейных модулей упругости блочных образцов полистирола, изготовленных по разной технологии, а также составных образцов, выполненных из 3 пластин полистирола, склеенных этилцианоакрилатным клеем. На основе полученных данных по изменению скорости ультразвуковых волн в образце в зависимости от приложенного статического давления были определены наборы модулей упругости третьего порядка (модулей Мурнагана) исследуемых образцов. Как известно, нелинейно-упругие параметры более чувствительны к структурным изменениям материала, чем линейные [3], поэтому технология изготовления полимерного материала может оказывать 
существенное влияние на значения нелинейно-упругих модулей.

\section{1. Материалы и экспериментальная методика}

В экспериментах использовались опытные образцы коммерческого сополимера стирола с диметакрилатом этиленгликоля (ДМЭГ) в концентрации 3 и 10\% (обозначены как $\mathrm{PS}_{\mathrm{comm} 3}$ и $\mathrm{PS}_{\text {comm10 }}$ соответственно), разработанные на Дзержинском заводе органического синтеза. Также использовались лабораторные образцы, изготовленные по расплавной технологии из гранулированного полистирола марки 585 (ОАО „Нижнекамскнефтехим“, Россия) с использованием микрокомпаундера DSM Xplore $5 \mathrm{ml}$ Microcompounder (DSM Xplore, Нидерланды). Часть образцов (обозначены как PSlab1) изготавливались путем переработки полистирола при температуре $200^{\circ} \mathrm{C}$ в течение $10 \mathrm{~min}$ и скорости вращения шнеков $\omega=120 \mathrm{~min}^{-1}$. Затем проводилось впрыскивание расплава полимера в прессформу, нагретую до $80^{\circ} \mathrm{C}$. Далее пресс-форма охлаждалась до комнатной температуры на воздухе. Более детально технология изготовления описана в работе [19]. Вторая часть образцов (обозначены как $\left.\mathrm{PS}_{\mathrm{lab} 2}\right)$ получалась переработкой полистирола при $280^{\circ} \mathrm{C}$ в течение $10 \mathrm{~min}$ и скорости вращения шнеков $\omega=120 \mathrm{~min}^{-1}$ с последующим литьем в пресс-форму, нагретую до $40^{\circ} \mathrm{C}$. Затем образец вынимался из прессформы. Лабораторные образцы изготавливались в виде пластин размером $1.5 \times 10 \times 50 \mathrm{~mm}$.

Поскольку лабораторные образцы были довольно тонкими, для увеличения площади соприкосновения образца с генератором и детектором ультразвуковых волн три такие пластины склеивались по наибольшей стороне этилцианоакрилатным клеем Супермомент. Выбор клея обусловлен тем, что, как было нами показано ранее $([20])$, он не приводит к изменению параметров нелинейных волн (солитонов) деформации в волноводах из полистирола, возможность формирования которых определяется нелинейными модулями упругости материала. В настоящей работе возможное влияние слоев клея на нелинейные модули упругости образца из полистирола было исследовано на склейке из трех пластин коммерческого сополимера.

Методика определения модулей упругости третьего порядка детально описана в нашей предыдущей работе [18] и предполагает измерение зависимости скорости распространения в образце трех типов ультразвуковых волн (продольных волн $v_{p}$ и поперечных волн с направлением колебаний перпендикулярно $v_{s \perp}$ и параллельно $v_{s} \|$ направлению давления) от величины приложенного к образцу статического давления $P$. Последующее определение коэффициентов наклона этих зависимостей $\left(\alpha_{x}, \alpha_{y}, \alpha_{z}\right)$ из полученных экспериментальных данных: $\rho v_{p}^{2}=\lambda+2 \mu+\alpha_{x} P ; \rho v_{s \|}^{2}=\mu+\alpha_{y} P ; \rho v_{p}^{2}=\mu+\alpha_{z} P$, и решение системы уравнений (1):

$$
\begin{gathered}
\alpha_{x}=-\frac{2 l-\frac{\lambda}{\mu}(2 m+\lambda+2 \mu)}{3 \lambda+2 \mu}, \\
\alpha_{y}=-\frac{m+\frac{\lambda}{4 \mu} n+2 \lambda+2 \mu}{3 \lambda+2 \mu}, \\
\alpha_{z}=-\frac{m-\frac{\lambda+\mu}{2 \mu} n-\lambda}{3 \lambda+2 \mu},
\end{gathered}
$$

позволяет определить набор из трех модулей упругости третьего порядка (модулей Мурнагана) $l, m, n$. Модули упругости второго порядка $\lambda$ и $\mu$ определяются из значений скоростей волн при нулевом давлении. Стоит отметить, что при приложении поперечного статического давления происходит не только изменение скорости распространения ультразвуковых волн, но и деформация самого образца. Это также может привести к увеличению длительности распространения ультразвуковых волн даже при неизменной скорости. Поэтому для корректного определения модулей упругости третьего порядка этот эффект был учтен при составлении системы уравнений (1). При этом в экспериментальной установке ультразвуковые преобразователи были прижаты к торцам образца и обеспечена возможность их сдвига при удлинении образца под воздействием внешнего давления.

Схема разработанной экспериментальной установки, используемой для определения модулей упругости третьего порядка, приведена на рис. 1, $a$. Исследуемый образец помещался в тиски с электронным динамометром АЦД/1С-20/1И-1, что позволяло изменять прикладываемую к образцу силу в диапазоне от 0 до $7 \mathrm{kN}$ с точностью $2 \mathrm{~N}$. Автоматическое считывание показателей динамометра и их синхронизация с измерением ультразвуковых волн в образце позволило автоматизировать эксперимент и ускорить обработку данных. Для создания равномерного давления к образцу с двух сторон прикладывались металлические блоки, покрывающие бо́льшую часть поверхности образца с двух сторон. Значения приложенного к образцу статического давления находились в диапазоне от 0 до $10 \mathrm{MPa}$, при этом не происходило необратимой деформации образца, а шаг повышения давления составлял около $1 \mathrm{MPa}$. Для генерации и детектирования продольных волн использовались ультразвуковые преобразователи P121 (Амати Акустика, Россия) с резонансной частотой $1.8 \mathrm{MHz}$, а генерация и детектирование поперечных волн производилась с помощью ультразвуковых преобразователей V154-RB (Olympus, CША) с резонансной частотой $2.25 \mathrm{MHz}$. Для того чтобы обеспечить возможность удлинения образца при повышении статического давления, ультразвуковые преобразователи, приложенные к торцам образца, не были жестко закреплены и могли раздвигаться на небольшое расстояние при приложении давления. Достаточно широкий спектральный диапазон 


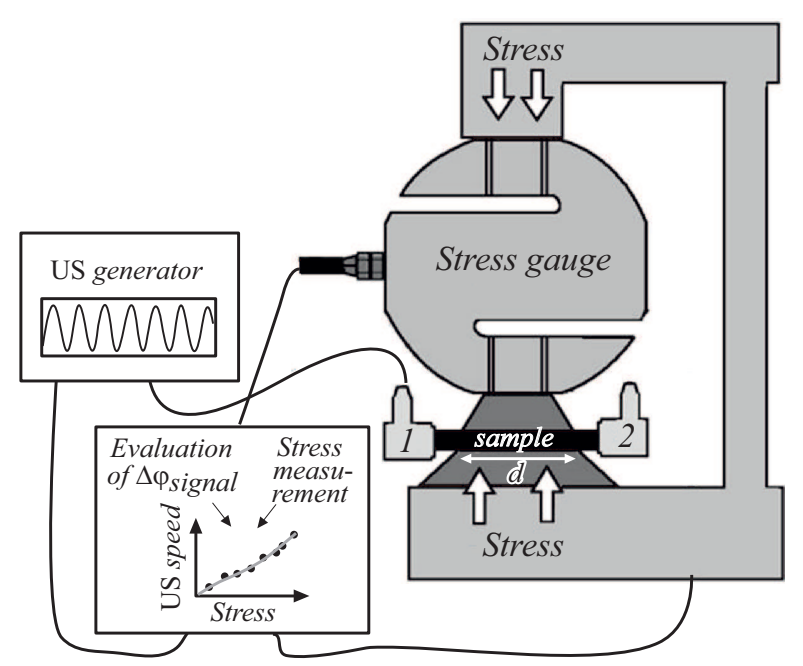

$a$

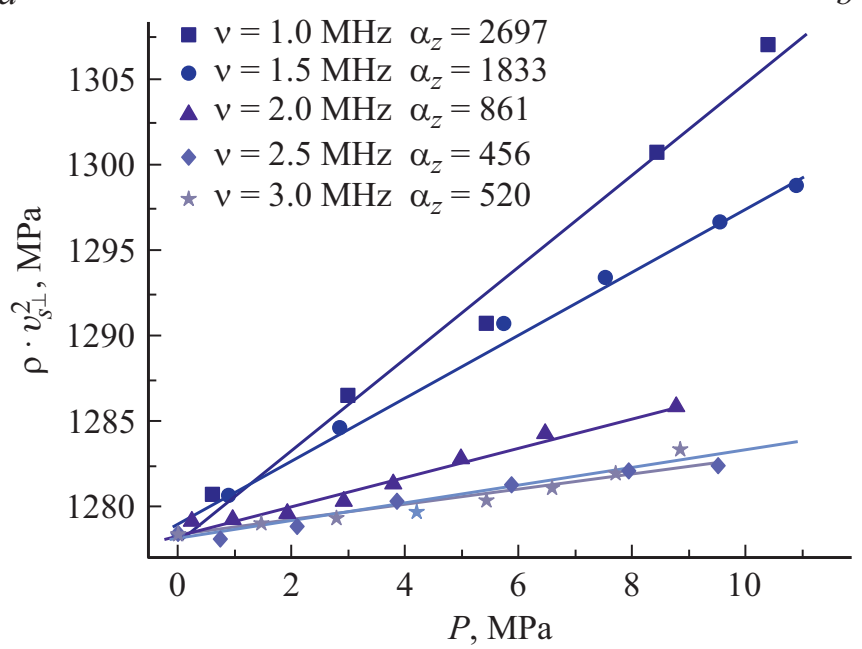

Рис. 1. Схема экспериментальной установки для измерения модулей упругости третьего порядка в образцах $(a)$, экспериментально измеренная зависимость $\rho v_{s \perp}^{2}(P)$ для поперечных волн, направленных перпендикулярно к оси приложенного давления при исследовании образца $\mathrm{PS}_{\mathrm{lab} 2}$ на разных частотах ультразвуковых волн $(b)$.

преобразования электрического сигнала в поперечные и продольные волны с помощью используемых преобразователей позволил проводить измерение скорости ультразвуковых волн в диапазоне частот от 1 до $3 \mathrm{MHz}$. Генерация синусоидальных сигналов с амплитудой $6 \mathrm{~V}$ производилась с помощью генератора AM300 Dual Arbitrary Generator (Rohde\& Schwarz), а детектирование и анализ экспериментальных данных осуществлялись с помощью осциллографа RTB2002 (Rohde\& Schwarz).

Для измерения скорости распространения звуковых волн при отсутствии давления и определения упругих модулей второго порядка исследуемый образец зажимался между ультразвуковыми преобразователями, на один из которых подавалась волна выбранной частоты с амплитудной модуляцией в виде прямоугольного сигнала. Анализ времени запаздывания между регистрируемым сигналом со второго ультразвукового преобразователя и синхроимпульсом позволял измерить время запаздывания и скорость распространения ультразвуковых волн на выбранной частоте. Измерение скорости распространения продольных и поперечных волн показало, что в диапазоне частот от 1 до $3 \mathrm{MHz}$ изменение скорости ультразвуковых волн при изменении частоты находится в пределах экспериментальной погрешности и модули упругости второго порядка могут быть приняты постоянными.

Для определения изменения скоростей поперечных и продольных ультразвуковых волн, возникающего при приложении внешнего статического давления к образцу, проводилась оценка относительного запаздывания ультразвуковых волн при наличии и отсутствии давления. При этом для проведения измерений скорости ультразвуковых волн на единственной выделенной частоте в качестве генерируемого сигнала использовался периодический синусоидальный сигнал. Это позволяло не только измерять зависимость скорости звука от давления на определенной частоте, но и избежать искажения формы исходного сигнала при различных давлениях и связанных с этим трудностей в последующей обработке экспериментальных данных. Идентичный синусоидальный сигнал, снимаемый со второго канала генератора сигналов, использовался для синхронизации и корректного определения времени запаздывания измеряемого сигнала. Кроме того, использование синусоидальных ультразвуковых волн позволило значительно ускорить процесс определения относительного запаздывания между сигналами, полученными при отсутствии $\left(\varphi_{0}\right)$ и наличии $\left(\varphi_{P}\right)$ статического давления величиной $P$. Для этого в каждом из зарегистрированных сигналов при статическом давлении $P$ определялась фаза синусоидальной волны $\varphi_{P}$. При этом синусоидальная волна, регистрируемая при отсутствии статического давления на образец $\varphi_{0}$, использовалась в качестве опорной величины, соответствующей предварительно измеренной скорости звука $v_{0}$. Таким образом, впоследствии разность фаз между двумя сигналами $\Delta \phi_{P}=\varphi_{P}-\varphi_{0}$ использовалась для вычисления времени задержки $\Delta t_{P}=\Delta \phi_{P} /(2 \pi f)$, где $f$ - частота генерируемого синусоидального сигнала, соответствуюшего изменению скорости распространения ультразвуковых волн. Для повышения точности определения относительной фазы детектируемого сигнала при каждом измерении совершалась регистрация 50 синусоидальных сигналов с их последующим усреднением. Принимая во внимание длину образца, к которой прикладывалось давление, $d=40 \mathrm{~mm}$, а также скорость распространения ультразвуковых волн при отсутствии давления $v_{0}$, из полученной величины относительного запаздывания $\Delta t_{P}$ можно вычислить скорость распространения ультразвуковых волн при приложенном давлении $v(P)=d v_{0} /\left(d+\Delta t_{P} v_{0}\right)$. Фитинг зависимости $\rho v^{2}(P)$ 
Таблица 1. Скорости продольных и поперечных ультразвуковых волн и модули упругости второго порядка в образцах полистирола

\begin{tabular}{l|c|c|c|c|c}
\hline & $\mathrm{PS}_{\text {comm3 }}$ & $\mathrm{PS}_{\text {comm10 }}$ & $\mathrm{PS}_{\text {comm10 layered }}$ & PS $_{\text {lab1 }}$ & PS $_{\text {lab2 }}$ \\
\cline { 2 - 6 } $\begin{array}{l}\text { Скорость продольных } \\
\text { волн, } v_{p}, \mathrm{~m} / \mathrm{s}\end{array}$ & $2252 \pm 5$ & $2280 \pm 5$ & $2278 \pm 5$ & $2235 \pm 5$ & $2093 \pm 5$ \\
\hline $\begin{array}{l}\text { Скорость поперечных } \\
\text { волн, } v_{s}, \mathrm{~m} / \mathrm{s}\end{array}$ & $1165 \pm 4$ & $1189 \pm 4$ & $1191 \pm 4$ & $1158 \pm 4$ & $1088 \pm 4$ \\
\hline$\rho, \mathrm{g} / \mathrm{cm}^{3}$ & 1.05 & 1.06 & 1.06 & 1.00 & 1.08 \\
\hline$E, \mathrm{GPa}, \rho v_{l}^{2}$ & $5.37 \pm 0.02$ & $5.51 \pm 0.02$ & $5.50 \pm 0.02$ & $4.99 \pm 0.02$ & $4.73 \pm 0.02$ \\
\hline$\mu, \mathrm{GPa}, \rho v_{s}^{2}$ & $1.44 \pm 0.01$ & $1.49 \pm 0.01$ & $1.50 \pm 0.01$ & $1.34 \pm 0.01$ & $1.28 \pm 0.01$
\end{tabular}

для разных типов ультразвуковых волн линейной функцией позволил определить величины $\alpha_{x}, \alpha_{y}, \alpha_{z}$. Оценка погрешностей измерения этих величин $\Delta \alpha_{x}, \Delta \alpha_{y}, \Delta \alpha_{z}$ была получена непосредственно из линейного фитинга зависимостей $\rho v^{2}(P)$, а погрешности определения самих модулей упругости третьего порядка вычислялись из значений $\Delta \alpha_{x}, \Delta \alpha_{y}, \Delta \alpha_{z}, \Delta \lambda$ и $\Delta \mu$ согласно закону распространения случайных ошибок при решении системы уравнений (1).

\section{2. Результаты и обсуждение}

Примеры измеренных зависимостей $\rho v_{s \perp}^{2}(P)$ для поперечных волн, направленных перпендикулярно к оси приложенного давления при исследовании образца $\mathrm{PS}_{\mathrm{lab} 2}$, представлены на рис. 1, b. Как видно из полученных данных, измерение зависимости $\rho v_{s \perp}^{2}(P)$ без дальнейшего определения значений модулей упругости уже демонстрирует заметную зависимость нелинейных упругих свойств материала от частоты ультразвуковых волн.

Вследствие невысокой эффективности генерации продольных и поперечных волн проведение измерений на образцах в виде тонких пластин затруднено. Поэтому для увеличения площади соприкосновения ультразвуковых преобразователей с торцами и получения надежного сигнала в таких образцах приходится осуществлять склейку нескольких пластин, выполненных из одного вида материала. При этом встает вопрос о влиянии такой склейки на измеряемые значения упругих модулей. Для определения этого влияния при склеивании пластин этилцианоакрилатным клеем Супермомент было изготовлено 2 образца коммерческого полистирола $\mathrm{PS}_{\text {comm10 }}$ с размерами $10 \times 10 \times 60 \mathrm{~mm}$ в виде цельного бруска и в виде склейки трех пластин с размерами $3 \times 10 \times 60 \mathrm{~mm}$. При этом для обеспечения целостности склеенных образцов приложение статического давления осуществлялось поперек граней склейки образцов так, чтобы это не могло привести к деламинации и искажению результатов. Результаты измерения упругих модулей второго и третьего порядков в этих образцах, приведенные в табл. 1 и на рис. 2., демонстрируют отсутствие значимых различий параметров у этих образцов. Полученные результаты дают возможность определения модулей упругости лабораторных образцов полистирола на основе измерений, выполненных на склейках пластин из этих материалов. Кроме того, эти результаты являются обоснованием возможности проведения исследований распространения объемных нелинейных волн деформации в ламинированных волноводах, выполненных из полистирола и нанокомпозитов на его основе (см. [19-22]).

Для повышения точности и оценки погрешностей в процессе измерений, проводилось по два измерения зависимости $\rho v^{2}(P)$ для каждого образца и каждого типа ультразвуковых волн. Статистический анализ полученных данных позволил оценить значения модулей упругости третьего порядка и их погрешности с достаточной точностью. Значения измеренных нелинейных модулей упругости для четырех исследованных образцов полистирола представлены на рис. 3 и в табл. 2. В табл. 1

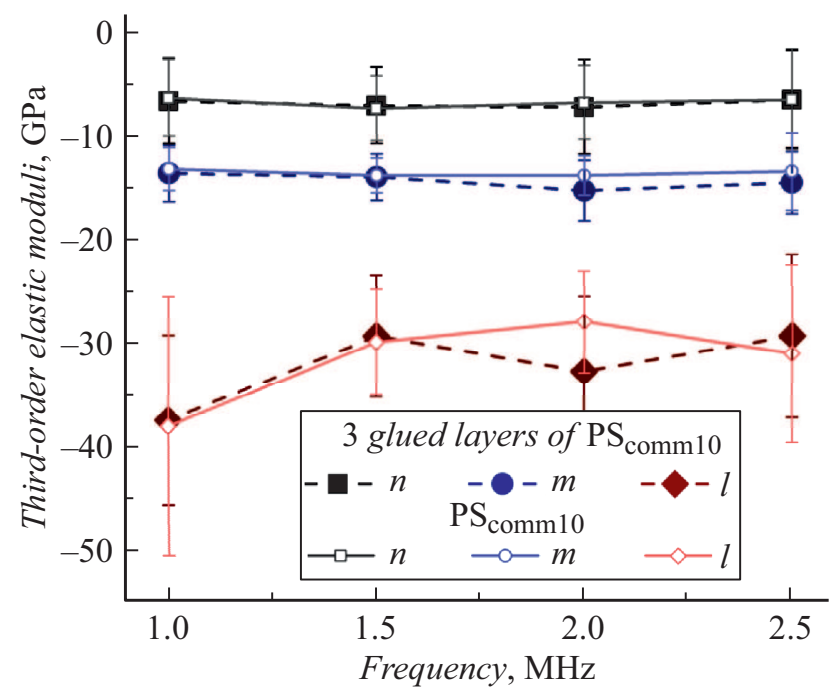

Рис. 2. Результаты измерения модулей упругости третьего

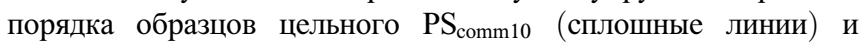
склейки из трех пластинок $\mathrm{PS}_{\mathrm{comm} 10}$ (штриховые линии). 

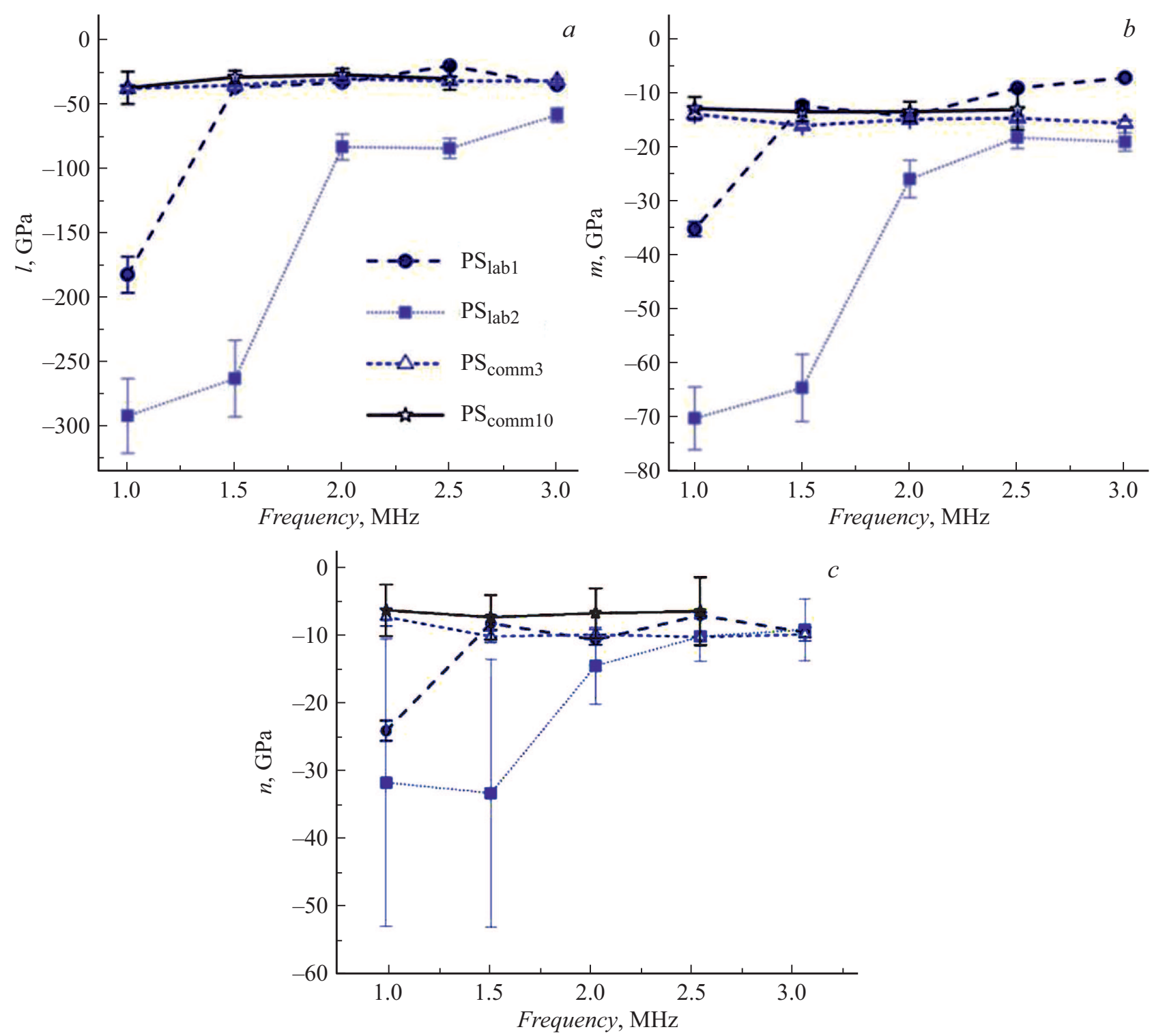

Рис. 3. Зависимости модулей упругости третьего порядка $l(a), m(b), n(c)$ от частоты ультразвуковых волн для образцов полистирола, изготовленных по разным технологиям. Исследуемые образцы указаны на рисунке $a$.

приведены также результаты определения плотности $\rho$, скорости продольных $v_{p}$ и поперечных $v_{s}$ волн, модуля Юнга $E$ и модуля сдвига $\mu$ (второго коэффициента Ламе) для этих образцов. Как показано на рис. 3, зависимость величин модулей упругости от частоты ультразвуковых волн существенно различна для образцов коммерческого сополимера стирола и лабораторных образцов полистирола. Все три модуля третьего порядка у двух образцов сополимера одинаковы в пределах погрешности и не зависят от частоты ультразвуковых волн в исследуемом диапазоне. У лабораторных образцов, напротив, наблюдается сильная зависимость модулей от частоты, в особенности на меньших частотах. Для образца $\mathrm{PS}_{\mathrm{lab} 1}$, полученного при переработке полистирола по первой технологии (смешение при температуре $200^{\circ} \mathrm{C}$, литье в нагретую до $80^{\circ} \mathrm{C}$ пресс-форму и ее охлаждение на воздухе), абсолютные значения модулей упругости третьего порядка на частоте $1 \mathrm{MHz}$ приблизительно в 3 раза больше, чем у сополимеров, а при более высоких частотах, начиная с $1.5 \mathrm{MHz}$, уменьшаются и выходят на плато, приближаясь к значениям у образцов сополимеров. В случае переработки полимера при более высокой температуре $\left(280^{\circ} \mathrm{C}\right)$ и литье в пресс-форму, нагретую до $40^{\circ} \mathrm{C}$ (образец $\left.\mathrm{PS}_{\mathrm{lab} 2}\right)$, частотная зависимость модулей упругости наблюдается практически во всем исследуемом диапазоне, причем абсолютные значения модулей значительно больше почти во всем диапазоне частот, что свидетельствует о более высокой нелинейности этого материала. При охлаждении полистирола он переходит в стекловидное состояние, которое является метастабильным, и его структура, а также температура стеклования зависят от скорости охлаждения полистиро- 
Таблица 2. Измеренные значения модулей упругости третьего порядка в образцах из полистирола

\begin{tabular}{|c|c|c|c|c|c|c|}
\hline \multirow{2}{*}{ Образец } & \multirow{2}{*}{ Модуль, GPa } & \multicolumn{5}{|c|}{ Частота ультразвуковых волн } \\
\hline & & $1 \mathrm{MHz}$ & $1.5 \mathrm{MHz}$ & $2 \mathrm{MHz}$ & $2.5 \mathrm{MHz}$ & $3 \mathrm{MHz}$ \\
\hline \multirow{3}{*}{$\mathrm{PS}_{\text {comm3 }}$} & $l$ & $-38.7 \pm 1.5$ & $-36.1 \pm 0.8$ & $-31.1 \pm 0.8$ & $-32.8 \pm 3.0$ & $-32.7 \pm 1.2$ \\
\hline & $m$ & $-13.2 \pm 2.0$ & $-13.8 \pm 1.7$ & $-13.8 \pm 1.8$ & $-14.9 \pm 0.4$ & $-15.9 \pm 0.6$ \\
\hline & $n$ & $-7.2 \pm 1.2$ & $-10.0 \pm 0.8$ & $-9.8 \pm 0.7$ & $-10.1 \pm 0.6$ & $-9.7 \pm 0.9$ \\
\hline \multirow{3}{*}{$\mathrm{PS}_{\mathrm{comm} 10}$} & $l$ & $-38.0 \pm 12$ & $-29.9 \pm 5.0$ & $-27.8 \pm 4.9$ & $-31.0 \pm 8.5$ & \\
\hline & $m$ & $-13.6 \pm 2.7$ & $-13.9 \pm 2.2$ & $-15.2 \pm 2.9$ & $-14.5 \pm 3.0$ & \\
\hline & $n$ & $-6.3 \pm 3.7$ & $-7.3 \pm 3.1$ & $-6.7 \pm 3.5$ & $-6.4 \pm 4.0$ & \\
\hline \multirow{3}{*}{$\begin{array}{r}\mathrm{PS}_{\text {comm10 }} \\
\text { layered }\end{array}$} & $l$ & $-37.5 \pm 8$ & $-29.3 \pm 5.8$ & $-32.7 \pm 7.2$ & $-29.3 \pm 7.9$ & \\
\hline & $m$ & $-13.6 \pm 2.7$ & $-13.9 \pm 2.2$ & $-15.2 \pm 2.9$ & $-14.5 \pm 3.0$ & \\
\hline & $n$ & $-6.6 \pm 4.1$ & $-7.0 \pm 3.7$ & $-7.2 \pm 4.4$ & $-6.5 \pm 4.6$ & \\
\hline \multirow{3}{*}{$\mathrm{PS}_{\mathrm{lab} 1}$} & $l$ & $-182 \pm 13$ & $-37.8 \pm 2.7$ & $-33.9 \pm 1.8$ & $-21.1 \pm 0.8$ & $-35.8 \pm 2.1$ \\
\hline & $m$ & $-35.5 \pm 1.3$ & $-12.7 \pm 0.8$ & $-14.6 \pm 0.7$ & $-9.4 \pm 2.8$ & $-7.5 \pm 0.4$ \\
\hline & $n$ & $-23.5 \pm 1.4$ & $-8.2 \pm 0.9$ & $-10.5 \pm 0.7$ & $-7.0 \pm 0.4$ & $-9.4 \pm 0.7$ \\
\hline \multirow{3}{*}{$\mathrm{PS}_{\mathrm{lab} 2}$} & $l$ & $-292 \pm 28$ & $-263 \pm 29$ & $-83.7 \pm 9.9$ & $-84.9 \pm 7.7$ & $-59.2 \pm 5.4$ \\
\hline & $m$ & $-72.9 \pm 5.8$ & $-64.9 \pm 6.2$ & $-26.2 \pm 3.5$ & $-18.5 \pm 2.0$ & $-19.4 \pm 1.7$ \\
\hline & $n$ & $-29.5 \pm 7.3$ & $-32.8 \pm 6.3$ & $-13.4 \pm 2.2$ & $-10.2 \pm 1.4$ & $-9.9 \pm 1.4$ \\
\hline
\end{tabular}

ла [23]. Поэтому более высокая нелинейность образцов может быть связана с тем, что при быстром охлаждении полимера формируется больший свободный объем для сегментальной подвижности полимерной цепочки, чем при медленном охлаждении на воздухе.

\section{Заключение}

Таким образом, в работе впервые были исследованы модули упругости третьего порядка образцов полистирола, изготовленных по разной технологии. Было показано, что изменение процентного содержания ДМЭГ в составе сополимера полистирола при концентрации до 10\% не оказывает влияния на значения нелинейных модулей упругости, однако приводит к небольшому увеличению модулей упругости второго порядка. В то же время показано, что изменение параметров технологического процесса изготовления материала может оказывать существенное влияние на его нелинейно-упругие характеристики, и даже небольшие изменения технологического процесса могут привести к изменениям структуры полимерного материала, которые хорошо заметны при измерении модулей упругости третьего порядка.

Показано, что наличие тонкого слоя этилцианоакрилатного клея не оказывает влияния на значения модулей упругости как второго, так и третьего порядка слоистого образца полистирола, что существенно при использовании слоистых пластиков. Полученные зависи- мости могут иметь большое практическое значение при использовании материалов при высоких динамических нагрузках.

\section{Благодарности}

Авторы благодарят В.Е. Юдина за предоставление оборудования для изготовления лабораторных образцов и за полезные обсуждения.

\section{Финансирование}

Работа выполнена при финансовой поддержке РНФ в рамках гранта № 17-72-20201.

\section{Конфликт интересов}

Авторы заявляют, что у них нет конфликта интересов.

\section{Список литературы}

[1] S. Takahashi, R. Motegi. SpringerPlus, 4 (1), 325 (2015). DOI: 10.1186/s40064-015-1019-2

[2] K.-Y. Jhang. Intern. J. Precision Engineer. Manufactur., 10 (1), 123 (2009).

[3] J.S. Valluri, K. Balasubramaniam, R.V. Prakash. Acta Mater., 58 (6), 2079 (2010).

[4] L.D. Landau, E.M. Lifshitz. Theory of Elasticity (Wiley, NY., 1956) 
[5] R.N. Thurston, K. Brugger. Phys. Rev., 133, A1604 (1964).

[6] E.D. Murnaghan. Finite Deformation of an Elastic Solid (Wiley, NY., 1951)

[7] J.K. Krüger, C. Grammes, K. Stockem, R. Zietz, M. Dettenmaier. Colloid and Polymer Sci., 269 (8), 764 (1991). DOI: 10.1007/BF00657442

[8] Ф.Е. Гарбузов, А.М. Самсонов, А.А. Семенов, А.Г. Шварц. Письма в ЖТФ, 42 (3), 16 (2016).

[9] C. Bermes. Appl. Phys. Lett., 90 (2), 021901 (2007). DOI: $10.1063 / 1.2431467$

[10] F. Masurkar, P. Tse. J. Phys. Commun., 3 (5), 055002 (2019). DOI: $10.1088 / 2399-6528 / a b 101 d$

[11] D.S. Hughes, J.L. Kelly. Phys. Rev., 92, 1145 (1953). DOI:10.1103/PhysRev.92.1145

[12] D.M. Egle, D.E. Bray. J. Acoust. Society of America, 60, 741 (1976).

[13] S. Takahashi. Mechan. Advanc. Mater. Modern Processes, 4, 2 (2018).

[14] А.И. Коробов, В.М. Прохоров, Д.М. Мехедов. ФТТ, 55 (1), 10 (2013).

[15] T. Bateman, W.P. Mason, H.J. McSkimin. J. Appl. Phys., 32, 928 (1961).

[16] Н.С. Аверкиев, Ю.В. Илисавский, В.М. Стернин. ФТТ, 29, 1450 (1987).

[17] K.W. Winkler, X. Liu. J. Acoust. Society of America, 100, 1392, (1996).

[18] A.V. Belashov, Y.M. Beltukov, O.A. Moskalyuk, I.V. Semenova. Polymer Testing, 95, 107132 (2021).

DOI: 10.1016/j.polymertesting.2021.107132

[19] O.A. Moskalyuk, A.V. Belashov, Y.M. Beltukov, E.M. Ivan'kova, E.N. Popova, I.V. Semenova, V.Y. Yelokhovsky, V.E. Yudin,. Polymers, 12, 2457 (2020). DOI:10.3390/polym 12112457

[20] G.V. Dreiden, K.R. Khusnutdinova, A.M. Samsonov, I.V. Semenova. J. Appl. Phys., 104, 086106 (2008). DOI: $10.1063 / 1.3000665$

[21] A.V. Belashov, Y.M. Beltukov, N.V. Petrov, A.M. Samsonov, I.V. Semenova. Appl. Phys. Lett., 112, 121903 (2018). DOI: $10.1063 / 1.5016944$

[22] G.V. Dreiden, K.R. Khusnutdinova, A.M. Samsonov, I.V. Semenova. J. Appl. Phys., 107, 034909 (2010). DOI: $10.1063 / 1.3294612$

[23] J.E.K. Schawe. Thermochimica Acta, 603, 128 (2015).

DOI: $10.1016 /$ j.tca.2014.05.025 